\title{
Estimación de la exposición intencionada a radiación ionizante en pacientes de una Unidad de Cuidados Intensivos Neonatales
}

\author{
Measurement of intentional exposure to ionizing radiation in a neonatal intensive care unit
}

\author{
Víctor Arízaga-Ballesteros, ${ }^{*, \ddagger}$ Rocío Garza-Castillo, ${ }^{*, \ddagger}$ María del Carmen Franco-Cabrera, ${ }^{\ddagger}, \S$ \\ Cristian Estrada-Hernández, ${ }^{\S}$ Víctor Javier Lara-Díaz, ${ }^{\ddagger}$ Mario René Alcorta-García*,‡ \\ * Departamento de Neonatología, Hospital Regional Materno Infantil, SSNL, Guadalupe, Nuevo León; ${ }^{\ddagger}$ Escuela \\ de Medicina y Ciencias de la Salud. Programa Multicéntrico de Especialidad Médica en Neonatología, Tecnológico \\ de Monterrey; ${ }^{\S}$ Seguridad Radiológica, Tecnofísica Radiológica, S.C., Monterrey, Nuevo León, México.
}

\section{RESUMEN}

Introducción: Las radiografías de tórax son de los procedimientos más frecuentes utilizados en las Unidades de Cuidados Intensivos Neonatales (UCIN). El objetivo de este estudio fue determinar la dosis de radiación por radiografía que recibieron los pacientes hospitalizados mediante el kerma en aire incidente (Ki), medida de la dosis de entrada en piel. Material y métodos: Estudio observacional, prospectivo, longitudinal y descriptivo. Se incluyeron pacientes atendidos en la UCIN del Hospital Materno Infantil, Nuevo León, México, a quienes se les tomó una o más radiografías toraco-abdominales (portátiles) durante su estancia. El Ki se midió de acuerdo por el Organismo Internacional de Energía Atómica para la dosimetría de pacientes pediátricos en radiografía simple. Resultados: Se analizaron 66 pacientes. En total se tomaron 416 radiografías, siendo la mediana por paciente de 13 (RIQ 2-26). El Ki fue de 140 (RIQ 80-450) $\mu$ Gy por radiografía. En $75 \%$ de las exposiciones, la mediana del Ki fue de $195 \mu \mathrm{Gy}$. Conclusiones: Las dosis de radiación por radiografías toraco-abdominales en recién nacidos en UCIN son mayores que las recomendadas.

Palabras clave: Radiación ionizante, neonato, pretérmino, Unidad de Cuidados Intensivos Neonatales, radiografías.

\begin{abstract}
Introduction: Chest $X$-rays are one of the most common procedures used in neonatal intensive care units (NICU). The objective of this study was to determine the $X$-ray radiation dose received by hospitalized patients by means of incident air kerma (Ki), a measure of the skin entry dose. Material and methods: Observational, prospective, longitudinal and descriptive study. We included patients treated at the NICU of the Hospital Materno Infantil, Nuevo León, Mexico, who had one or more thoraco-abdominal (portable) radiographs taken during their stay. Ki was measured according to the International Atomic Energy Agency for the dosimetry of pediatric patients in plain radiography. Results: Sixty-six patients were analyzed. In total, 416 radiographs were taken; the median number of radiographs per patient was 13 (IQR 2-26). The Ki was 140 (IQR 80-450) $\mu$ Gy by radiography. In $75 \%$ of the exposures, the median Ki was $195 \mu$ Gy. Conclusions: In newborns cared for in the NICU, the radiation doses from thoraco-abdominal radiographs are higher than international recommendations.
\end{abstract}

Keywords: lonizing radiation, newborn, preterm, Neonatal Intensive Care Unit, $x$-rays.

Correspondencia: Mario René Alcorta-García, E-mail: mario.alcorta@tec.mx

Citar como: Arízaga-Ballesteros V, Garza-Castillo R, Franco-Cabrera MC, Estrada-Hernández C, Lara-Díaz VJ, Alcorta-García MR. Estimación de la exposición intencionada a radiación ionizante en pacientes de una Unidad de Cuidados Intensivos Neonatales. Rev Mex Pediatr. 2021; 88(3): 96-100. https://dx.doi.org/10.35366/102189 


\section{INTRODUCCIÓN}

Los estudios radiológicos, particularmente la radiografía de tórax (incluyendo su variante más común en Neonatología, la proyección toraco-abdominal), son uno de los procedimientos más realizados en la Unidad de Cuidados Intensivos Neonatales (UCIN). ${ }^{1}$

La exposición a la radiación ionizante asociada con los estudios radiográficos puede tener efectos adversos para la salud, entre los que se encuentran los defectos hereditarios y la inducción de neoplasias. ${ }^{2}$ Estos efectos adversos dependen del tiempo de exposición y de la dosis recibida. ${ }^{3}$ Dado el riesgo derivado de la alta frecuencia de estudios radiográficos que habitualmente se realizan en pacientes bajo cuidados intensivos neonatales, particularmente los neonatos prematuros, se han recomendado precauciones específicas. ${ }^{4}$

Es conocido que la radiosensibilidad de los tejidos aumenta con la tasa de división celular, la cual es alta en prematuros y en recién nacidos ( $\mathrm{RN})$; además, se ha establecido que el riesgo de daño por radiación es directamente proporcional a la dosis recibida. ${ }^{5,6}$

Para determinar el balance riesgo/beneficio parece necesario medir la cantidad de radiación que reciben los pacientes, ${ }^{7}$ como se ha hecho en Perú en $2013,{ }^{8}$ y en la Ciudad de México en $2012 .{ }^{9}$ El objetivo de este estudio fue determinar la dosis de radiación que recibieron los pacientes hospitalizados en la UCIN y compararla con los niveles de referencia.

\section{MATERIAL Y MÉTODOS}

Estudio observacional, prospectivo, longitudinal y descriptivo. Fue realizado en el Hospital Regional Materno Infantil, el cual es un hospital de tercer nivel perteneciente a los Servicios de Salud de Nuevo León, localizado en Ciudad Guadalupe (área metropolitana de Monterrey). Este hospital cuenta con 75 camas en neonatología y 25 camas en la UCIN, el hospital atiende en promedio 1,500 nacimientos por mes.

En el estudio se incluyeron neonatos internados en la UCIN que requirieron más de una radiografía durante su estancia. Todas las radiografías del estudio se tomaron con equipo portátil, marca GE Medical Systems, modelo 2275938, Milwaukee, Wisconsin, USA. Las imágenes se procesaron en un lector de CR marca Carestream, modelo Classic, Rochester, NY, USA.

Debido a las condiciones clínicas de los pacientes y a la indicación de los tratantes, las proyecciones fueron toraco-abdominal, en decúbito dorsal, tomadas con el paciente dentro de una incubadora o en una cuna térmica. En la UCIN hay separación entre las camas de alrededor de 1.5 metros a los lados, y tres metros al frente entre cada cama. Esta distancia entre camas es considerada suficiente para evitar la exposición inadvertida de radiación entre pacientes al momento de toma de una radiografía. ${ }^{1}$

La colección de los datos se realizó del 1 de abril al 30 de junio de 2016. El indicador de la radiación recibida por el paciente seleccionado fue el kerma en aire incidente (Ki), equivalente a la dosis de entrada en piel, cuya unidad de medición es el micro Gray ( $\mu \mathrm{Gy}$ ). Estos datos, permiten comparar nuestros resultados con el nivel de referencia establecido en las Guías Europeas de Calidad en Radiografía Pediátrica para la proyección radiográfica, AP de tórax neonatal con equipo portátil, $80 \mu \mathrm{Gy}$ de Ki en superficie de entrada. ${ }^{10,11}$

El Ki se determinó mediante el método recomendado por el Organismo Internacional de Energía Atómica para la dosimetría de pacientes pediátricos en radiografía simple (International Atomic Energy Agency 2013). 9,11,12

La determinación del Ki se obtuvo con la medición del haz de rayos-X utilizando la siguiente ecuación:

$$
K i=Y(d) P i t\left(\frac{d}{D F S D}\right) 2
$$

Donde: $Y(d)$ es el rendimiento del tubo de rayos-X medido a una distancia del punto focal, con el kilovoltaje $(\mathrm{kVc})$ y filtración total empleada para la radiografía de los pacientes. $P_{i t}$ es el producto corriente tiempo también conocido como mAs, utilizado en la radiografía de cada paciente. $D_{F S D}$ es la distancia del punto focal a la superficie del paciente donde entra el rayo central del haz, que es igual a la diferencia en la distancia focoreceptor de imagen y el espesor del paciente.

En cada radiografía se registró el kilovoltaje $(\mathrm{kVc})$, producto corriente tiempo (mAs) y la distancia focoreceptor de imagen $\left(D_{F S D}\right)$. El rendimiento del tubo de rayos-X se midió a cada valor de $\mathrm{kVc}$ en el registro en los equipos portátiles de radiografía disponibles en el hospital. Con esos datos se obtuvo el valor de $Y(d)$ para cada radiografía. La medición se realizó con un medidor de radiación de estado sólido (marca RTI, modelo Piranha) con certificado de calibración vigente.

También se incluyó la fecha de la toma, el peso del $\mathrm{RN}$, el espesor del tórax del paciente y el número de radiografías realizadas; estos datos fueron tomados por los médicos del estudio, personal de enfermería de la UCIN y personal técnico de radiología. 
Tabla 1: Variables demográficas de los 66 recién nacidos evaluados.

\begin{tabular}{lccc} 
Variable & $\mathrm{n}(\%)$ & Mín. & Máx. \\
Masculino & $34(51.5)$ & & \\
Femenino & $32(48.5)$ & & \\
Edad gestacional (semanas) & 32 & 24 & 41 \\
Peso (kg)* & 2.44 & 0.68 & 4.1 \\
Estancia en UCIN (días) & 58 & 3 & 113 \\
Radiografías (n) & 13 & 1 & 26 \\
\hline & & & \\
UCIN = Unidad de Cuidados Intensivos Neonatales. & & \\
${ }^{*}$ Mediana. &
\end{tabular}

Análisis estadístico. El análisis fue de tipo descriptivo. Las variables continuas se expresan como medianas y rango intercuartilar (RIQ) porque, basado en la prueba de Kolmogorov-Smirnov, no tenían distribución normal. Las variables cualitativas se presentan con frecuencia absoluta y porcentajes.

El protocolo fue aprobado por los Comités de Ética e Investigación del Tecnológico de Monterrey y del Hospital Materno Infantil de Alta Especialidad.

\section{RESULTADOS}

En el periodo de estudio se admitieron a la UCIN 160 pacientes. Sólo se analizaron los datos en $66 \mathrm{RN}$ a los que se les realizó más de una radiografía. El resto no se incluyó porque durante su estancia se tomó una o ninguna radiografía.

Los datos demográficos y antropométricos de los 66 pacientes estudiados se describen en la Tabla 1; poco más de la mitad fueron masculinos, $80 \%$ fueron a pretérmino y $20 \%$ a término. El total de radiografías tomadas a este grupo fue de 416 estudios. La mediana de radiografías tomadas por paciente fue de 13 (RIQ 2-26).

Respecto al voltaje $(\mathrm{kVc})$ la mediana fue 62.0 (RIQ 60.0-68.0). Mientras que la mediana del producto corriente-tiempo (mAs) fue de 2.0 (RIQ 2.0-2.5). En cuanto al Ki, la mediana fue de 140 (RIQ $80-450) \mu \mathrm{Gy}$ por radiografía (Tabla 2).

Cincuenta por ciento de las radiografías emplearon 120-140 $\mu$ Gy en una exposición de rayos-X. El percentil 75 de Ki (que representa $75 \%$ de las radiografías tomadas) fue de $195 \mu \mathrm{Gy}$, lo que nos permite inferir que por lo menos en $25 \%$ de los estudios (104 eventos) se emplearon valores superiores a los recomendados.

\section{DISCUSIÓN}

Para garantizar que la exposición a la radiación sea segura, es necesario conocer la dosis usada en la práctica cotidiana. Es muy importante que la toma de las radiografías sea ajustada al área a evaluar, a este respecto se ha sugerido que la toma de radiografías toraco-abdominales no es conveniente, puesto que en éstas el foco de rayos $\mathrm{X}$ se dirige en un área más amplia, en detrimento de la calidad del estudio. En nuestro estudio ese aspecto no se logró controlar; sin embargo, refleja la práctica habitual en el contexto del trabajo cotidiano.

Como lo documentamos en el presente estudio, el número de radiografías de tórax puede considerarse alto. En las guías clínicas basadas en evidencia se señala que la indicación para su realización debe ser razonada para evitar las radiografías de "rutina", lo cual tiene como meta disminuir la exposición injustificada a los rayos X. En la información presentada, no llegamos a discernir si los estudios efectuados estaban justificados o no.

Por otro lado, es posible ajustar los valores de emisión de los aparatos de rayos X portátiles sin detrimento de la calidad de imagen, este punto es también muy importante. ${ }^{1,13}$ Más recientemente, la utilización de la ultrasonografía de manera rutinaria en la UCIN representa una oportunidad muy valiosa para disminuir la exposición a los rayos X. ${ }^{14}$

Se comparó la Ki, representativa de la dosis de entrada en piel, con los niveles de referencia establecidos en Estados Unidos de Norteamérica (EUA) y en la Comunidad Europea. Las dosis de nuestro estudio fueron evidentemente mayores. El nivel de referencia descrito en las Guías Europeas de Radiografía pediátrica para la proyección AP de tórax neonatal con equipo portátil es de $80 \mu \mathrm{Gy}$ de Ki. ${ }^{10}$

El percentil 75 de Ki de este estudio es de interés porque sería el mejor estimador del nivel habitual de

Tabla 2: Valores de equipos de radiología portátil utilizados.

\begin{tabular}{lccc} 
& Mediana & Mín. & Máx. \\
kVc & 65 & 50 & 80 \\
mAs & 2.28 & 1.84 & 2.72 \\
Ki $(\mu \mathrm{Gy})$ & 243.8 & 65 & 422.6 \\
\hline
\end{tabular}

$\mathrm{kVc}=$ kilovoltaje; $\mathrm{mAs}=$ miliamperios por segundo; $\mathrm{Ki}(\mu \mathrm{Gy})=$ kerma incidente (micro Grays). 
exposición y, por tanto, el nivel de referencia de dosis local. ${ }^{11}$ En este estudio el percentil 75 del Ki fue de $195 \mu \mathrm{Gy}$, más del doble del valor de referencia y sólo comparado a lo reportado por Fletcher y colaboradores en un hospital del Reino Unido en $1986,{ }^{15}$ y más del doble de lo reportado por Frayre y su equipo en la Ciudad de México en $2012{ }^{9}$ y Sharma y su grupo en 2018 en India. ${ }^{16}$ Además, es $>40 \%$ de lo reportado en el estudio de Paniagua y colaboradores en Perú, en 2013. ${ }^{8}$ Cabe aclarar que el Ki fue calculado con base en los valores obtenidos de los aparatos de radiología; en otros estudios se obtuvo con base en los valores detectados, mediante dosímetros de termoluminiscencia, que no son la mejor forma de medir la exposición, lo cual hace a nuestro estudio más cercano a la realidad.

Las Guías Europeas de Calidad en Radiografía Pediátrica (1996) establecieron el nivel de referencia en $80 \mu \mathrm{Gy}$ para la radiografía neonatal. ${ }^{10} \mathrm{El}$ Colegio Americano de Radiología tiene como nivel de referencia $60 \mu \mathrm{Gy}$ para un paciente con tórax de $12.5 \mathrm{~cm}$ de espesor. ${ }^{14}$

El Ki en nuestro estudio es alto en referencia al espesor y peso de los pacientes observados. La causa parece ser la selección de valores altos de $\mathrm{kV}$ y mAs en los ajustes del equipo portátil. Para un mismo paciente, el voltaje empleado varió desde 50 hasta $80 \mathrm{kV}$. De la revisión de las técnicas radiográficas registradas durante el estudio, encontramos que aquéllas que resultan en un Ki comparable al nivel de referencia $(78 \mu \mathrm{Gy})$ emplearon un voltaje de $58 \mathrm{kV}$ y producto tiempo corriente de $1.6 \mathrm{mAs}$, cifras que caen dentro de las recomendaciones internacionales. ${ }^{15,17}$

Es conveniente recordar que la exposición a rayos $\mathrm{X}$ se asocia a un riesgo de carcinogénesis, cuya probabilidad es proporcional a la dosis recibida. En cuanto al uso seguro, las autoridades científicas recomiendan la vigilancia continua de la dosis y la implementación de medidas que garanticen que el riesgo sea razonablemente bajo, en comparación con el beneficio. ${ }^{10,17}$

Por otro lado, en centros que utilizan radiografía digital, se ha reportado que la dosis por radiografía es mayor que los niveles de referencia establecidos en EUA y Europa. ${ }^{1,18}$ El uso de radiografía digital favorece que pase desapercibida la amplia variación en la dosis de entrada. Si se usara película radiográfica sería evidente, porque daría como resultado imágenes sobreexpuestas o "quemadas". Este incremento inadvertido de la dosis es un fenómeno bien reconocido cuando se migra de película a radiografía digital, ${ }^{1,19,20}$ y eso precisamente ocurrió en nuestro hospital el año previo a nuestro estudio.
El beneficio de la exposición a radiación debe ser mayor que el riesgo, haciendo un uso inteligente de la radiación. En cuanto a la optimización, se refiere a aplicar el principio de ALARA (as low as reasonably achievable). ${ }^{13,18}$

En revisiones previas se ha evidenciado la amplia variabilidad en cuanto a las dosis a las que son expuestos los neonatos en la UCIN, ${ }^{21,22}$ por lo que se han propuesto diversas estrategias para disminuir la exposición, tales como la estandarización de proceso, ${ }^{23}$ o el registro de la dosis de radiación. ${ }^{24}$ La Academia Americana de Pediatría sugirió la protección de los pacientes durante los procedimientos para disminuir el riesgo. ${ }^{25}$

En concordancia con las recomendaciones ya comentadas, los resultados del presente estudio fueron presentados en el mismo hospital, lo cual hizo posible reducir la dosis por radiografía en los pacientes de la UCIN. Esto se logró mediante la colaboración interdepartamental para establecer políticas y procedimientos de garantía de calidad. Estas acciones incluyeron la estandarización de las técnicas radiográficas, la vigilancia del buen funcionamiento de los equipos de rayos $\mathrm{X}$, el monitoreo de la dosis y la evaluación de la justificación de las órdenes médicas de estudios de diagnóstico.

\section{CONCLUSIONES}

La evaluación de la radiación por exposición a radiografías en RN parece ser un procedimiento que debería formar parte de los procesos de evaluación de la calidad de toda UCIN, a fin de garantizar la seguridad de los pacientes.

\section{AGRADECIMIENTOS}

Expresamos nuestra gratitud al personal de enfermería de la UCIN del Hospital Regional Materno Infantil, por su apoyo durante la realización de este trabajo.

\section{REFERENCIAS}

1. Hinojosa-Armendáriz VI, Mejía-Rosales SJ, Franco-Cabrera MC. Optimization of radiation dose and image quality in mobile neonatal chest radiography. Radiography. 2018; 24(2): 104-109.

2. Scott MV, Fujii AM, Behrman RH, Dillon JE. Diagnostic ionizing radiation exposure in premature patients. J Perinatol. 2014; 34(5): 392-395.

3. da Costa Pinto F, Rocha G, Flor-de-Lima F, Soares H, Ramos I, Guimaraes H. Radiation exposure in very low birth weight infants. Minerva Pediatr. 2019; 71(3): 247-252 
4. Kartikeswar GAP, Parikh TB, Pandya D, Pandit A. lonizing radiation exposure in NICU. Indian J Pediatr [Internet]. 2020; 87(2): 158-160.

5. Furlow B. Radiation protection in pediatric imaging. Radio/ Technol. 2011; 82(5): 421-439.

6. Toma P, Bartoloni A, Salerno S, Granata C, Cannatà V, Magistrelli $A$ et al. Protecting sensitive patient groups from imaging using ionizing radiation: effects during pregnancy, in fetal life and childhood. Radiol Med. 2019; 124(8): 736-744.

7. Don S. Radiosensitivity of children: potential for overexposure in $\mathrm{CR}$ and $\mathrm{DR}$ and magnitude of doses in ordinary radiographic examinations. Pediatr Radiol. 2004; 34(S3): S167-S172.

8. Paniagua Muños RP. Estimación de dosis en exámenes de rayos $X$ para neonatos en el Hospital Nacional Dos de Mayo [Tesis]. Perú: Universidad Nacional del Callao; 2013. Disponible en: http://repositorio.unac.edu.pe/bitstream/ handle/UNAC/142/RolinsonPaul_Tesis_tituloprofesional_2013. pdf? sequence $=3$ \&isAllowed $=y$

9. Frayre AS, Torres P, Gaona E, Rivera T, Franco J, Molina N. Radiation dose reduction in a neonatal intensive care unit in computed radiography. Appl Radiat Isot. 2012; 71 Suppl: 57-60.

10. Directorate-General XII: Science R and D, A. European Guidelines on Quality Criteria for Diagnostic Radiographic Images [Internet]. 1996. p. 88. Available in: https://www.sprmn.pt/pdf/ EuropeanGuidelineseur16260.pdf

11. Huda W. Kerma-area product in diagnostic radiology. Am J Roentgenol. 2014; 203(6): W565-W569.

12. International Atomic Energy Agency (IAEA). Radiation protection in Paediatric Radiology, Safety reports series no. 71, IAEA, Vienna. IAEA Saf Reports Ser. 2012; 71: 128.

13. Willis CE, Slovis TL. The ALARA concept in pediatric CR and DR: Dose reduction in pediatric radiographic exams - A white paper conference Executive Summary. Pediatr Radiol. 2004; 34 (suppl. 3): 2004-2005.

14. Kurepa D, Zaghloul N, WatKins L, Liu J. Neonatal lung ultrasound exam guidelines. J Perinatol . 2018; 38(1): 11-22.

15. Fletcher EWL, Baum JD, Draper $\mathrm{G}$. The risk of diagnostic radiation of the newborn. Br J Radiol. 1986; 59(698): 165-170.

16. Sharma J, Sarma J, Agarwal S. Assessment of diagnostic reference level in radiography of neonatal chest anteroposterior examination: a hospital-based study. J Med Phys. 2018; 43(3): 200-203.

17. Vassileva J, Rehani MM, Applegate K, Ahmed NA, Al-Dhuhli H, Al-Naemi HM. IAEA survey of paediatric computed tomography practice in 40 countries in Asia, Europe, Latin America and Africa: procedures and protocols. Eur Radiol. 2013; 23(3): 623-631.

18. Aramesh M, Zanganeh KA, Dehdashtian M, MaleKian A, Fatahiasl $\mathrm{J}$. Evaluation of radiation dose received by premature neonates admitted to neonatal intensive care unit. J Clin Med Res. 2017; 9(2): 124-129.

19. Chawla SC, Federman N, Zhang D, Nagata K, NuthakKi S, McNittGray $\mathrm{M}$ et al. Estimated cumulative radiation dose from PET/CT in children with malignancies: a 5-year retrospective review. Pediatr Radiol. 2010; 40(5): 681-686.

20. Amis ES, Butler PF, Applegate KE, Birnbaum SB, Brateman LF, Hevezi JM et al. American College of Radiology white paper on radiation dose in medicine. J Am Coll Radiol [Internet]. 2007; 4(5): 272-2784.

21. Yu CC. Radiation safety in the Neonatal Intensive Care Unit: too little or too much concern? Pediatr Neonatol. 2010; 51(6):311-319.

22. Gordon PV, Swanson JR. A simple step to reduce radiation exposure in the NICU. J Perinatol. 2014; 34(5): 331-332.

23. Seibert JA, Morin RL. The standardized exposure index for digital radiography: An opportunity for optimization of radiation dose to the pediatric population. Pediatr Radiol. 2011; 41(5): 573-581.

24. Makri T, YakoumaKis E, Papadopoulou D, Gialousis G, Theodoropoulos V, Sandilos $P$ et al. Radiation risk assessment in neonatal radiographic examinations of the chest and abdomen: a clinical and Monte Carlo dosimetry study. Phys Med Biol. 2006; 51(19): 5023-5033.

25. Risk of ionizing radiation exposure to children: a subject review. American Academy of Pediatrics. Committee on Environmental Health. Pediatrics. 1998; 101(4 Pt 1): 717-719.

Conflicto de intereses: los autores declaran que no tienen. 